\title{
Endoscopic screening of relatives of patients with colorectal cancer
}

\author{
L M Hunt, P S Rooney, J D Hardcastle, N C Armitage
}

\begin{abstract}
Background-The risk of colorectal cancer is higher among relatives of those affected. The neoplastic yield reported from screening such individuals varies enormously between studies and depends on the age and strength of the family history of those screened.

Aims-To ascertain the neoplastic yield of endoscopic screening of first degree relatives of patients with colorectal cancer by age and familial risk.
\end{abstract}

Subjects-A total of 330 individuals with a family history of colorectal cancer.

Method-Endoscopic screening conducted according to a protocol.

Results-Adenomas were found in $12 \%$, and adenomas larger than $1 \mathrm{~cm}$ in $8 \%$, of "high risk" individuals screened primarily by colonoscopy. Of those with neoplasia, $26 \%$ had lesions at or proximal to the splenic flexure. Neoplasia was found in $\mathbf{9 . 5 \%}$ of individuals at lower familial risk, screened primarily by $60 \mathrm{~cm}$ flexible sigmoidoscopy, $4 \%$ of whom had neoplasia larger than $1 \mathrm{~cm}$ in size or cancer. Neoplastic yield was greatest in the fourth and fifth decades in those at highest risk, but increased with age in those at lower risk.

Conclusions-For individuals with two or more first degree relatives, or relatives who have developed colorectal cancer at a young age, colonoscopy appears to be the only satisfactory method of screening, but $60 \mathrm{~cm}$ flexible sigmoidoscopy may be useful in those at lower levels of risk. (Gut 1998;42:71-75)

Keywords: colorectal cancer; endoscopic screening; colonoscopy; sigmoidoscopy; familial risk

Department of Surgery, University of Nottingham, Queens Medical Centre, Nottingham L M Hunt

P S Rooney

J D Hardcastle

N C Armitage

Correspondence to: Dr N C Armitage, Department of Surgery, Floor E, West Block, University Hospital, Queens Medical Centre, Nottingham NG7 2UH, UK.

Accepted for publication 2 May 1997
Neoplastic yield reported from endoscopic screening of relatives of patients with colorectal cancer varies enormously between studies. Retrospective reports of colonoscopy give rates
Table 1 Screening protocol

\begin{tabular}{ll}
\hline Affected relatives & Screening modality \\
\hline $\begin{array}{l}\text { First degree }<50 \text { years or }>2 \text { first } \\
\text { degree or first degree plus second } \\
\text { degree }<50 \text { years }\end{array}$ & $\begin{array}{l}\text { Colonoscopy } \\
\text { First degree or first and second }\end{array}$ \\
$\begin{array}{l}\text { Faecal occult blood test } \\
\text { degree }>50 \text { years }\end{array}$ & $\begin{array}{l}\text { plus } 60 \mathrm{~cm} \text { flexible } \\
\text { sigmoidoscopy }\end{array}$ \\
\hline
\end{tabular}

of polyp detection as diverse as 10.6 and $63 \% .{ }^{15}$ Adenoma yield in prospective studies is generally lower, varying between 12 and $22 \%$ using colonoscopy ${ }^{1314}{ }^{17}$ and 5.3 and $21 \%$ using $60 \mathrm{~cm}$ flexible sigmoidoscopy. ${ }^{18}{ }^{19}$ This disparity results not only from differences in study design, but also the age and strength of the family history of the individuals screened. Many reports fail to give relevant information, particularly on the latter issue. This makes evaluation of the potential benefit of screening difficult and has led to conflicting screening recommendations for individuals at risk.

\section{Patients and methods}

Individuals were recruited for screening via an open access clinic, hospital referral, and case finding initiatives, which were running for 28 months from June 1991. Individuals were counselled before screening about their familial risk and the type of screening being recommended. No individuals had had previous colorectal neoplasia, inflammatory bowel disease, or familial adenomatous polyposis. These individuals were not involved in other screening initiatives in Nottingham during that time period. ${ }^{20}$ Screening was conducted according to a protocol (table 1$)$. Individuals with positive faecal occult blood tests or neoplasia detected on $60 \mathrm{~cm}$ flexible sigmoidoscopy were screened by total colonoscopy regardless of familial risk. Colonoscopy was complete to the caecum in 165 of $193(85 \%)$ individuals undergoing colonoscopy as a primary screening procedure. Twenty six individuals also had a completion barium enema. Two individuals failed to attend for barium enema. The $60 \mathrm{~cm}$ flexible sigmoidoscopy was carried out without sedation. Mean depth of insertion of the sigmoidoscope was $57 \mathrm{~cm}$, and in $91(66 \%)$ individuals the splenic flexure was definitively identified.

Screening was recommended from the age of 40 years, except in families where there was evidence of hereditary non-polyposis colorectal cancer, or where a relative had developed cancer at a young age, when screening was recommended from the age of 20 years, or five years before the youngest affected relative whichever was the earlier. Screening results were collected prospectively and stored on a computer 
Table 2 Colonoscopic screening of individuals from families with three or more first degree relatives with colorectal cancer (1:2 risk)

\begin{tabular}{lccl}
\hline Age $(y)$ & No screened (\%) & No with neoplasia (\%) & No with neoplasia $>$ 1cm (\%) \\
\hline$<20$ & $4(4)$ & $1(25)$ & $1(25)$ \\
$20-29$ & $16(15)$ & $1(6)$ & 0 \\
$30-39$ & $21(19)$ & $4(19)$ & $4(19)$ \\
$40-49$ & $27(25)$ & $4(15)$ & $3(11)$ \\
$50-59$ & $24(22)$ & 0 & 0 \\
$60-69$ & $14(13)$ & $2(14)$ & $1(7)$ \\
$>70$ & $4(4)$ & $2(50)$ & 0 \\
Total & 110 & $14(13)$ & $9(8)$ \\
\hline
\end{tabular}

Table 3 Colonoscopic screening of individuals with two first degree relatives with colorectal cancer or a relative affected under the age of 50 years (1:6-1:10 risk)

\begin{tabular}{llll}
\hline Age $(y)$ & No screened (\%) & $\begin{array}{l}\text { No with adenoma } \\
(\%)\end{array}$ & $\begin{array}{l}\text { No with adenoma }>1 \text { cm or severe } \\
\text { dysplasia (\%) }\end{array}$ \\
\hline$<20$ & $4(5)$ & $1(25)$ & $1(25)$ \\
$20-29$ & $12(14)$ & $1(8)$ & 0 \\
$30-39$ & $15(18)$ & 0 & 0 \\
$40-49$ & $20(24)$ & 0 & 0 \\
$50-59$ & $15(18)$ & $2(13)$ & $2(13)$ \\
$60-69$ & $17(20)$ & $5(29)$ & $4(24)$ \\
Total & $83(100)$ & $9(11)$ & $7(8)$ \\
\hline
\end{tabular}

database. Results were analysed by age and family risk. Full data were available on 330 individuals (202 women and 128 men).

\section{Results}

Table 2 shows the results of screening 110 individuals (median age 44 (range 17-74) years) from families with at least three first degree relatives with colorectal cancer (1:2 risk). The histological findings were as follows. Ten individuals had tubular adenomas, two had tubulovillous adenomas, and two had unusual large dysplastic/metaplastic polyps. (Other metaplastic polyps were excluded from the analysis.) Twelve patients had a single lesion, one had two lesions, and one had more than ten. The site of the lesion was as follows: three in the right colon (at or proximal to the splenic flexure); five in the left colon; five in the rectum; one in the left colon and rectum. Adenomas larger than $1 \mathrm{~cm}$ were found in the right colon (one), the left colon (four), and the rectum (four). The yield of neoplasia over $1 \mathrm{~cm}$ in size was highest in individuals in their fourth and fifth decades, and this fell over the age of 50 years. Ten individuals were referred because of symptoms or a combination of a family history of colorectal cancer and symptoms. Of this subgroup, one had an adenoma detected. Sixteen individuals admitted to symptoms on enquiry for which they had not sought medical advice. Adenomas were detected in two, and in one of these it was larger than $1 \mathrm{~cm}$.

Table 3 shows the results of screening 83 individuals (median age 45 (range 18-75)

Table $460 \mathrm{~cm}$ flexible sigmoidoscopy screening of individuals with a single first degree, or first and second degree relatives, with colorectal cancer over the age of 50 years (1:12-1:17 risk)

\begin{tabular}{lclll}
\hline Age $(y)$ & No screened (\%) & $\begin{array}{l}\text { No with cancer } \\
(\%)\end{array}$ & $\begin{array}{l}\text { No with adenoma } \\
(\%)\end{array}$ & $\begin{array}{l}\text { No with neoplasia }>1 c m \\
(\%)\end{array}$ \\
\hline $20-29$ & $7(5)$ & & $1(4)$ & \\
$30-39$ & $25(18)$ & & $2(5)$ & $1(3)$ \\
$40-49$ & $37(27)$ & & $4(11)$ & $1(3)$ \\
$50-59$ & $38(27)$ & $2(8)$ & $2(33)$ & $1(17)$ \\
$60-69$ & $24(18)$ & & $11(8)$ & $5(4)$ \\
$>70$ & $6(4)$ & $2(1.5)$ & & \\
Total & 137 & & & \\
\hline
\end{tabular}

years) with two first degree relatives with colorectal cancer, a first degree relative with colorectal cancer under the age of 50 years, or both a first and second degree relative with the disease, one of whom was under the age of 50 years (1:6-1:10 risk). Histological findings were as follows. Six individuals had tubular adenomas, one had a tubulovillous adenoma, one had a villous adenoma, and one a tubular adenoma plus a tubulovillous adenoma. Four adenomas in three individuals were severely dysplastic, three of which were $5 \mathrm{~mm}$ tubular adenomas. Five individuals had a single lesion, three had two, and one had five. The site of the lesion was as follows: two in the right colon, two in the left colon, three in the rectum, one in the right colon and rectum, and one in the left colon and rectum. The site of adenomas larger than $1 \mathrm{~cm}$ or with severe dysplasia was as follows: two in the right colon, one in the left colon, two in the rectum, one in the right colon and rectum, and one in the left colon and rectum. Eight individuals were referred because of symptoms or a combination of a family history of colorectal cancer and symptoms. Of this subgroup, one had neoplasia $(>1 \mathrm{~cm})$. Twenty three individuals had symptoms on enquiry for which they had not sought medical advice. Three had adenomas, and two of these were larger than $1 \mathrm{~cm}$.

Yield by family type was as follows: two of 26 individuals with a single first degree relative under the age of 50 years (mean age of subgroup 42 (range 22-67) years) had an adenoma larger than $1 \mathrm{~cm}$ or exhibited severe dysplasia. One of 29 individuals with a first and second degree relative affected, one of whom was under 50 years (mean age of subgroup 37 (range 18-61) years) had an adenoma larger than $1 \mathrm{~cm}$. Five of 17 individuals who had two first degree relatives who developed colorectal cancer after the age of 50 years (mean age of subgroup 61 (range 42-75) years) had adenomas (four larger than $1 \mathrm{~cm}$ or with severe dysplasia). One of 11 individuals with both parents affected with colorectal neoplasia (mean age of subgroup 43 (range 28-61) years) had a $5 \mathrm{~mm}$ adenoma.

Table 4 shows the results of screening of 137 individuals (median age 49 (range 24-75) years) with a single first degree, or first and second degree relative, with colorectal cancer over the age of 50 years (1:12-1:17 risk). Two individuals had cancer (rectal polyp cancer and Duke's type B sigmoid), one had tubulovillous adenoma, and ten had tubular adenomas. Nine patients had a single lesion, one had two, and one had more than five adenomas. The site of neoplasia was as follows: four in the left colon; six in the rectum, and one in the left colon and rectum. No additional right sided pathology was detected on colonoscopy in those with distal neoplasia. Twenty one individuals were referred because of symptoms or a combination of family history and symptoms. Of this subgroup, three had neoplasia (two $>1 \mathrm{~cm}$ ). Twenty four individuals had symptoms on enquiry for which they had not sought medical advice. Four had neoplasia (two $>1 \mathrm{~cm}$ ). 
Table 5 Pathological features in 36 individuals found to have neoplasia at endoscopy

\begin{tabular}{lclc}
\hline & $1: 2$ & $1: 6-1: 10$ & $1: 12-1: 17$ \\
\hline Multiple adenomas $>4$ & 1 & 1 & 1 \\
Severe dysplasia & 2 & 3 & 0 \\
Adenomas >1cm & 9 & 4 & 5 \\
Proximal neoplasia only & 3 & 2 & NA \\
Distal neoplasia only & 11 & 6 & NA \\
Proximal and distal neoplasia & 0 & 1 & 2 \\
Cancer & 0 & 0 & 5 \\
Single <5mm adenoma & 5 & 2 & 13 \\
Total & 14 & 9 &
\end{tabular}

NA, not applicable.

Table 5 gives a summary of the pathological features in 36 individuals found to have neoplasia at endoscopy.

\section{Discussion}

The yield of neoplasia (12\%) among those screened primarily by colonoscopy is notable because of their young age. All these individuals may be considered to be at "high risk" of colorectal cancer (lifetime risks of colorectal cancer of $1: 2$ to $1: 10) .^{7}$ To date prospective studies of colonoscopic screening in individuals with two or more affected first degree relatives (1:2 and 1:6 risk) have generally been small. Yield has ranged from $24 \%$ of 21 individuals $^{21}$ to $36 \%$ of 18 and 39 individuals. ${ }^{14}{ }^{18}$ Stephenson $e t$ al ${ }^{9}$ found adenomas in $10 \%$ of 20 people with either two or more relatives affected or a relative younger than 45 years affected (1:2-1:10 risk). A large report found neoplasia in $19.5 \%$ of 202 individuals with three or more affected relatives (1:2 risk) and in $11 \%$ of 132 individuals with two first degree relatives or a relative aged under 45 years (1:6-1:10 risk). This work is difficult to evaluate as adenoma size and age of those screened are not given. ${ }^{8}$ However, an update ${ }^{17}$ found adenomas in $27 \%$ of individuals from hereditary non-polyposis colorectal cancer families, $9 \%$ having adenomas that were "significant" (>1 cm/villous component/ moderately dysplastic). Adenomas were found in $21 \%$ of other individuals at $1: 10$ or greater risk, with 5\% having "significant" lesions.

We found that, among those with three or more relatives affected (1:2 risk), neoplastic yield was highest in the fourth and fifth decades of life. A fifth of individuals aged 30-39 had adenomas over $1 \mathrm{~cm}$ in size. Yield then fell over the age of 50 years. Although about a fifth of cancers in individuals with hereditary nonpolyposis colorectal cancer present over the age $60,{ }^{22-24}$ many of those surviving to later life without developing colorectal cancer are those who have not in fact inherited the mutant gene, and are therefore not at high risk of colorectal cancer. Age is more difficult to evaluate for those with two first degree relatives or relative(s) affected at a young age (1:6-1:10 risk). We found the incidence of neoplasia to be higher over the age of 50 years in this group, suggesting environmental causes. This is supported by the fact that all of the $29 \%$ of individuals with two older first degree relatives affected found to have neoplasia were themselves over the age of 50. However, a proportion of this group had neoplasia below the age of 30 years. Screening studies, including ours, tend to group these individuals together. ${ }^{89}$ This simplifies screening protocols and the presentation of results. However, these individuals are a heterogeneous group. Some, although ascribed a 1:10 risk, have unrecognised hereditary non-polyposis colorectal cancer (for instance where a parent is affected by a new mutation or is illegitimate). Others, where several relatives have developed colorectal cancer late in life, may be subject to shared environmental factors. A bimodal age distribution of neoplasia has previously been observed, ${ }^{14}$ but our numbers are not large enough to draw conclusions. The relatively low yield of neoplasia observed in this group as a whole means that many individuals were screened unnecessarily. A higher yield could be achieved by postponing screening. However, until those individuals who are the relatives of hereditary non-polyposis colorectal cancer patients can be reliably distinguished from those who have developed "sporadic" disease at an unusually young age, such a policy will miss lesions in the small proportion of individuals with unrecognised hereditary nonpolyposis colorectal cancer. The possibility of mutational analysis in index patients and predictive testing of relatives will enable a more refined screening strategy in the future.

Yield obtained using screening $60 \mathrm{~cm}$ flexible sigmoidoscopy varies between published series. Yield of adenomas in individuals with a single affected relative (1:17 risk) ranges from $5.3 \%$ (58\% of adenomas being less than 5 $\mathrm{mm}$ in size $)^{18}$ to $17 \%$ ( $8 \%$ larger than $1 \mathrm{~cm}$ in size). ${ }^{9}$ An adenoma yield from flexible sigmoidoscopy of $19 \%$ has been reported. ${ }^{25}$ However, despite the impressive size of this study ( $\mathrm{n}$ $=670$ ), over a quarter of those screened were from one family making evaluation difficult. The yield of total colonoscopy in individuals at this level of risk has also varied between 13 and $19 \%$ (mean age 55 and 51 respectively). ${ }^{21}{ }^{14} \mathrm{We}$ found neoplasia in $9.5 \%$ of those screened primarily by $60 \mathrm{~cm}$ flexible sigmoidoscopy, $4 \%$ of whom had neoplasia larger than $1 \mathrm{~cm}$ in size. These individuals are not considered to be at high risk of colorectal cancer; most have only a single relative affected in later life. However, the yield of neoplasia is high, given the relatively young age of those screened. The prevalence of both sporadic colorectal cancer and adenomas increases with age. ${ }^{26}$ We have observed a similar pattern in individuals at lower levels of familial risk. Neoplastic yield, among those with a single older first degree, or first and second degree relatives with colorectal cancer, increased steadily from $4 \%$ of those in their fourth decade to $33 \%$ of those over the age of 70 years. In studies in which yield has been broken down by age, findings have been similar to our own, with the incidence of neoplasia rising from $0-4.3 \%$ under the age of 40 years to $16-32 \%$ over the age of 60 years. ${ }^{13} 151821$ Comparing yield with that found in the general population is important, particularly at lower levels of familial risk. Yield obtained with $60 \mathrm{~cm}$ flexible sigmoidoscopy was $5.7 \%$ in a population of mean age 55 years. ${ }^{27}$ Although all controlled 
studies have shown a higher yield in family members than controls, , $^{9182125}$ even in one of the larger studies this has failed to reach statistical significance. ${ }^{21}$

Neoplastic yield among high risk individuals referred for screening because of symptoms was lower than that in those recruited by other methods. At first sight this appears surprising; however, a possible explanation lies in the fact that many of these individuals were initially seen in a general surgical clinic, where those with obvious pathology or particularly suspicious symptoms were investigated directly rather than being referred for familial screening. The presence of symptoms on enquiry for which medical attention had not been sought appears to be a poor indicator of neoplasia in those at very high risk of colorectal cancer. However, at lower levels of familial risk, more neoplasia was detected in those referred with symptoms than in the group as a whole. Furthermore, $17 \%$ of individuals who admitted to symptoms on enquiry for which they had not sought medical advice had neoplasia, $8 \%$ having neoplasia over $1 \mathrm{~cm}$ in size. Some $6 \%$ of asymptomatic individuals had neoplasia, only $1 \%$ having neoplasia larger than $1 \mathrm{~cm}$. This difference is not statistically significant but it may be that the presence of symptoms in individuals at lower levels of family risk can help to discriminate those more likely to have pathology. Certainly, the presence of symptoms in those with a family history of colorectal cancer should always be taken seriously.

We found that, among individuals from families with three or more affected first degree relatives (1:2 risk), $21 \%$ of adenomas and $11 \%$ of adenomas larger than $1 \mathrm{~cm}$ in size were at or proximal to the splenic flexure. Among individuals with two affected first degree relatives or a relative affected at a young age (1:6-1:10 risk), $33 \%$ of adenomas and $43 \%$ of adenomas larger than $1 \mathrm{~cm}$ or exhibiting severe dysplasia were proximal to the splenic flexure. Some $29 \%$ of individuals had isolated proximal neoplasia over $1 \mathrm{~cm}$ in size or had severe dysplasia. Similar findings were observed in high risk individuals screened at St Mark's Hospital, London, where $40 \%$ of adenomas in individuals at $1: 2$ risk and $43 \%$ of adenomas in individuals at 1:6-1:10 risk were proximal. ${ }^{8}$ These findings show that left sided endoscopy is an unsatisfactory method of screening these families and support the use of full colonoscopy as the screening modality of choice for such individuals.

Colonoscopy in expert hands is now a safe procedure and it is possible that it may be the screening modality of choice at all levels of familial risk. Indeed, there are those who currently advocate colonoscopy for all levels of familial risk. However, this recommendation is largely based on retrospective data analysis of individuals of unknown familial risk. ${ }^{11}$ Retrospective analyses have reported isolated proximal polyps in up to one third of individuals undergoing colonoscopy because of familial risk. ${ }^{12} 1628$ However, the extent of familial risk in those screened is not stated. This makes evaluation of these results difficult as many of those screened may have hereditary nonpolyposis colorectal cancer. Significantly, prospective studies have found isolated proximal neoplasia in only $1.5-5 \%$ of those screened. ${ }^{913} 29$ One controlled study found $48 \%$ of adenomas in relatives $(88 \%$ of whom had only a single first degree relative affected) were beyond the splenic flexure, compared with $25 \%$ in controls. However, this difference was not statistically significant, the size of the adenomas is not stated, it is unclear whether these were isolated proximal lesions, or how many individuals were affected. ${ }^{21}$ Therefore in itself this study is not sufficient to make a recomendation of colonoscopy for all levels of familial risk. Most proximal neoplasms detected by screening are small tubular adenomas similar to those found at post-mortem examination. ${ }^{30-34}$ The capacity of these lesions to progress to cancer is not established, and the site of an adenoma may itself influence the chance of progression to cancer. ${ }^{33}{ }^{34}$ We found no additional proximal neoplasia among individuals at lower levels of familial risk, who underwent colonoscopy consequent on a positive result from flexible sigmoidoscopy. Further follow up is required before the efficacy of 60 $\mathrm{cm}$ flexible sigmoidoscopy in these individuals is known, but initial data and those of other studies $^{91329}$ suggest that it may be useful and should be further explored. As shared environmental factors probably contribute to the development of colorectal cancer in many families, ${ }^{3}$ a distribution of cancer similar to that in sporadic disease is not unexpected. It is important to remember that, to be of any value, screening recomendations for those at risk of familial colorectal cancer must be pragmatic. LMH was supported by a locally funded research grant from
Trent Regional Health Authority. P S R was supported by the Cancer Research Campaign.

1 Stephenson BM, Finan PJ, Gascoyne J, et al. Frequency of familial colorectal cancer. Br f Surg 1991;78:1162-6.

2 Dumilial colorectal cancer. Br F Surg 1991;78:1162-6. rectum and colon in North-East Scotland. Gut 1982;23: rectum and 169-7

3 Lovett E. Family studies in cancers of the colon and rectum. Br f Surg 1976;63:13-18

4 St. John DJB, McDermott FT, Hopper JL, et al. Cancer risk in relatives of patients with common colorectal cancer. Ann Intern Med 1993;118:785-90.

5 Kee F, Collins BJ. How prevalent is cancer family syndrome? Gut 1991;32:509-12.

6 Mecklin J-P. Frequency of hereditary colorectal cancer. Gastroenterology 1987;93:1021-5.

7 Slack J. Family cancer syndromes. $\mathcal{F} R$ Soc Med 1989;82: 233-4.

8 Houlston R.S, Murday V, Haracopos C, et al. Screening and genetic counselling for relatives of patients with colorectal cancer in a family cancer clinic. BMf 1990;301:366-8.

9 Stephenson BM, Murday VA, Finan PJ, et al. Feasibility of family based screening for colorectal neoplasia: experience in one general surgical practice. Gut 1993;34:96-100.

10 Stevenson GW, Hernandez C. Single visit screening and treatment of first degree relatives: colon cancer pilot study. Dis Colon Rectum 1991;34:1120-4.

11 Dunlop MG. Screening for large bowel neoplasms in individuals with a family history of colorectal cancer. $\operatorname{Br} \mathcal{F}$ Surg 1992;79:488-94.

12 Herrera L, Hanna S, Petrelli N, Nava H. Screening endoscopy in patients with family history positive $(\mathrm{FH}+)$ for colorectal neoplasia (CRN). Gastrointest endosc 1990;36: 105 [abstract].

13 McConnell JC, Nizen JS, Slade MS. Colonoscopy in patients with a primary family history of colon cancer. Dis Colon Rect 1990;33:105-7.

14 Orrum WJ, Brzezinski WS, Weins EW. Hereditary colorectal cancer: a prospective, community based endoscopic study. Dis Colon Rectum 1990;33:490-3.

15 Luchtefeld MA, Severn D, Solfelt M, et al. Is colonoscopic screening appropriate in asymptomatic patients with family history of colon cancer? Dis Colon Rect 1991;43:763-8. 
16 Gryska PV, Cohen AM. Screening asymptomatic individuals at high risk for colon cancer with full colonoscopy. Dis als at high risk for colon canc

17 Gaglia P, Atkin WS, Whitelaw S, et al. Variables associated with the risk of colorectal adenomas in asymptomatic patients with a family history of colorectal cancer. Gut 1995;36:385-90.

18 Rozen P, Fireman Z, Figer A, et al. Family history of colorectal cancer as a marker of potential malignancy within a screening program. Cancer 1987;60:248-54.

19 Burt RW, Bishop T, Cannon LA, et al. Dominan inheritance of colonic polyps and colorectal cancer. N Engl $\mathcal{7}$ Med 1985;312:1540

20 Hardcastle JD, Thomas WM, Chamberlain J, et al. Randomised controlled trial of faecal occult blood screening for colorectal cancer. Lancet $1989 ; \mathbf{i}: 1160-4$.

21 Guillem JG, Forde KA, Treat MR, et al. Colonoscopic screening for neoplasms in asymptomatic first degree rela-
tives of colon cancer patients. Dis Colon Rect 1992;35:523tives
9 .
Vasen
Hit 22 Vasen HFA, den Hartog Jager FCA, Menko FH, Nagengast
FM. Screening for hereditary nonpolyposis colorectal cancer: a study of 22 kindreds in the Netherlands. $A m f$ Med 1989;86:278-81.

23 Fitzgibbons RJ, Lynch HT, Stanislav GV. Recognition and treatment of patients with hereditary non-polyposis colon cancer (Lynch syndromes 1 and 11). Ann Surg 1987;206: 289-95.

24 Mecklin JP, Jarvinen HJ. Clinical features of colorectal carcinoma in cancer family syndrome. Dis Colon Rectum 1986, 29:160-4.
25 Cannon-Albright LA, Skolnick MH, Bishop DT, et al. Common inheritance of susceptibility to colonic adenomatous polyps and associated colorectal cancers. N Engl F Med 1988;319:533-7.

26 Gillespie PE, Chambers TJ, Chan KW, et al. Colonic adenomas: a colonoscopic survey. Gut 1979;20:240-5.

27 Wherry DC, Thomas WM. The yield of flexible fiberoptic sigmoidoscopy in the detection of asymptomatic colorectal neoplasia. Surg Endosc 1994;8:279-81.

28 Guillem JG, Neugut AI, Forde KA, et al. Colonic neoplasms in asymptomatic first-degree relatives of colorectal cancer patients. Am f Gastroenterol 1988;83:271-3.

29 McConnell JC. Colonoscopy or flexible proctosigmoidoscopy. Dis Colon Rectum 1990;33:722.

30 Winawer SJ, Zauber A, Diaz B. The national polyp study: overview of programme and preliminary report of patient
and polyp characteristics. In: Steele G, Burt WR, and polyp characteristics. In: Steele G, Burt WR, Winanwer SJ, eds. Basic and clinical perspectives of colorectal
polyps and cancer. New York: Alan R Liss, 1988:35-49.

31 Williams AR, Balasooriya BAW, Day DW. Polyps and cancer of the large bowel: a necropsy study in Liverpool. Gut 1982;23:835-42.

32 Bombi JA. Polyps of the colon in Barcelona, Spain. An autopsy study. Cancer 1988;61:1472-6.

33 Konishi F, Morson BC. Pathology of colorectal adenomas: a colonoscopic survey. $\mathcal{f}$ Clin Pathol 1982;35:830-41.

34 Eide TJ. The age-, sex- and site-specific occurrence of adenomas and carcinomas of the large intestine within a defined population. Scand f Gastroenterol 1986;21:1083. 